\title{
Toward the reflectance measurement of micro components
}

\author{
Hervé Piombini \\ herve.piombini@cea.fr \\ Philippe Voarino \\ Dominique Breider \\ Fabien Lemarchand
}

\author{
CEA, DAM, Le Ripault, F-37260 Monts, France \\ QOL, 13 Avenue Louise Michel. 28500 Vernouillet, France \\ Institut de Recherches R. Bosch, 1027 Lonay, Suisse, France \\ Institut Fresnel, Unité Mixte de Recherche 6133, Université Paul Cezanne, 13397 Marseille Cedex 20, \\ France
}

Today, the controls by reflection of optical coatings are most of the time made with flat samples thanks to commercial spectrophotometers. However, components become smaller and more complex, curvature radius of lenses or mirrors are larger, so that measures are not so accurate and sometimes are not possible. Flat samples don't represent anymore the real reflection ability of the component. So to perform this kind of measurements, special devices are needed. A new means developed by the French Atomic Energy Commission (CEA) is proposed to fill in this gap. This device has a accuracy of $0.06 \%$ on flat sample over the $400 \mathrm{~nm}$ to $950 \mathrm{~nm}$ wavelength range with a spot size of $100 \mu \mathrm{m}$. It can measure the reflectance of samples even if their shapes are spherical. We investigate stainless steel balls and optical micro components (mirrors and lens) thanks to the tiny size of the analyzing spot of our reflectometer. Herein we introduce our first results on small optical components and show the limiting factors of our device. [DOI: 10.2971/jeos.2010.10034s]

Keywords: reflectometer, micro component, reflectance, spherical component, coating

\section{INTRODUCTION}

The controls by optical means of coatings deposited on optical components are generally made with flat witnesses thanks to commercial spectrophotometers. With flat and thin samples, spectrophotometers measure accurately transmission but reflectance factor measurements exhibit larger uncertainties [1]. Moreover when the components are spherical or aspherical, like lenses or mirrors, the spectral response can vary because of the non uniformity of thickness that is really linked to the deposition process and their position in the coating chamber [2]. For large radius of curvature, control can be achieved even with classical spectrophotometers. However, control becomes more and more difficult even impossible [3] when the radius of curvature decreases or when the optical device has a complex shape such as slicers for example [4]. So to perform this kind of measurement, special devices are needed [5]-[7]. For the optical micro components which are increasingly used in laser optical systems because of their new and many industrial applications, optical characterisations are very hard due to their shape and small size.

A new device $[6,8]$ to measure reflectance has been built by the French Atomic Energy Commission (CEA). It can measure the reflectance of samples even if their shapes are spherical [9]. This device has a good accuracy on flat samples [10]. Herein, we investigate samples made of stainless steel having various curvature radii to prove the obtained results with this device are the same whatever the sample shape may be.
We also demonstrate that we can measure optical micro components (mirrors and lens) thanks to the tiny size of the analyzing spot of our reflectometer on the sample. Our first results on small optical components are presented in this paper.

\section{THE USED REFLECTOMETER}

The reflectometer was designed to improve the accuracy of the reflectance measurement. This device has already been patented [6] and presented in various conferences and reviews [2, 8]-[11]. This experimental set-up is schematically drawn in Figure 1 and summarized below.

This device is designed to measure the specular reflectance of mirrors over the $400 \mathrm{~nm}$ to $950 \mathrm{~nm}$ wavelength range. The light beam is conditioned to enter the monochromator that selects the wavelength. The signal is spatially filtered and time modulated. The spatial filter defines the diameter of the spot (about $100 \mu \mathrm{m}$ ) on the surface of sample because the two points are conjugated. A set of long pass filters held in a wheel absorbs the parasitic harmonics of the monochromator. Two lock-in amplifiers are tuned to the frequency of the chopper. They allow acquisition of modulated signals but not ambient noises. Then, the beam is divided into two paths, one for reference and another for measurement. The sample mounts depend on the form and dimension of the sample. Different holders are put on a magnetic kinematic base to obtain good 


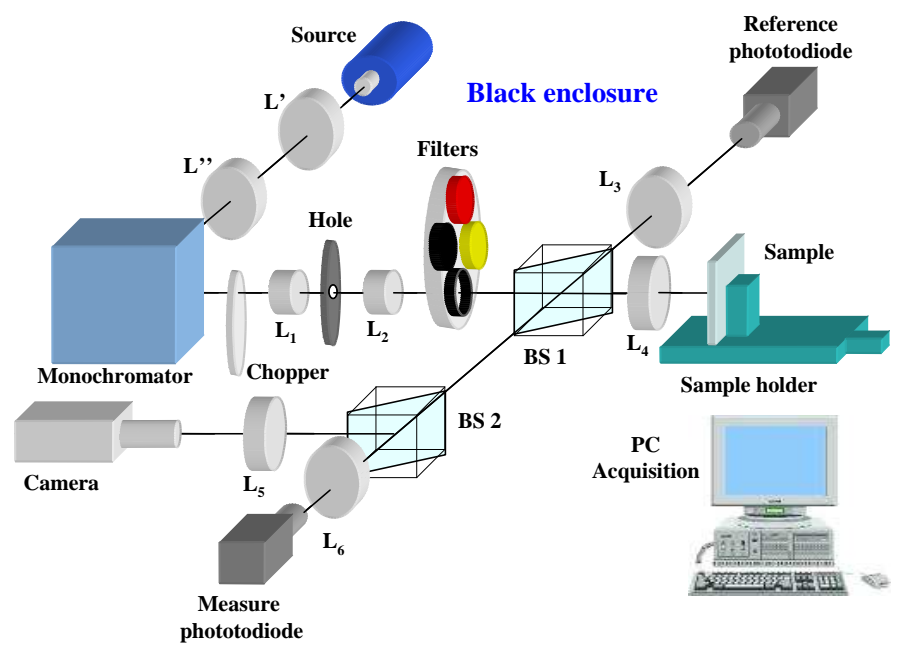

FIG. 1 Experimental set-up of CEA device.

repositioning. A magnetic mounting is fastened onto a manual goniometer, as a manual rotation stage is necessary to point correctly to the sample. This assemblage is fastened on a motorized three axes translation stage. It enables mappings to be obtained ( $y$ - and $z$-stages) and automatically conjugates ( $x$-stage) the photodiode with the surface of sample using the video path. As soon as the conjugation is achieved, the signals are acquired by the lock-in amplifiers for several seconds in order to make an average and to reject values outside the range defined by three times the standard deviation. By calculating the ratio between the measurement channel and the reference channel, problems due to the fluctuations of the light source power are reduced. This procedure is repeated for each wavelength. The reflectance factor is given by

$$
R(\lambda)=K(\lambda) \frac{V_{\text {Measurement }}(\lambda)-V_{\text {Measurement background }}(\lambda)}{V_{\text {Reference }}(\lambda)-V_{\text {Reference background }}(\lambda)}
$$

where $K(\lambda)$ is a calibration constant, $V_{\text {Measurement is }}$ the output voltage of the measurement photodiode,

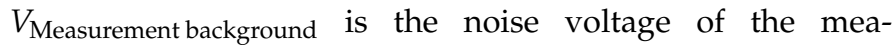
surement photodiode, $V_{\text {Reference }}$ is the output voltage of the reference photodiode, $V_{\text {Reference background }}$ is the noise voltage of the reference photodiode, and $\lambda$ is the wavelength. This procedure is repeated for each wavelength. To calibrate the measuring system in order to know $K(\lambda)$, a set of high reflectivity dielectric mirrors is used.

The software, which controls the device, enables it to focus the beam automatically on the surface of the sample at each wavelength, allowing a more accurate result, thanks to a very good repeatability [2]. The reproducibility of the focusing process is presented in Figure 2. To measure it, the component was randomly positioned, placed along the optical $x$-axis. A procedure of automatic focusing was made at $633 \mathrm{~nm}$, and then the location was recorded. This process was restarted several times, and each focalization was compared with the average position that was calculated from all the measurements. The value, which corresponds to twice the standard deviation $(\sigma)$ of the measurement, is $6 \mu \mathrm{m}$.

The characteristic of our device is to be able to measure shaped parts $[9,11]$ because we use a condenser lens with very large

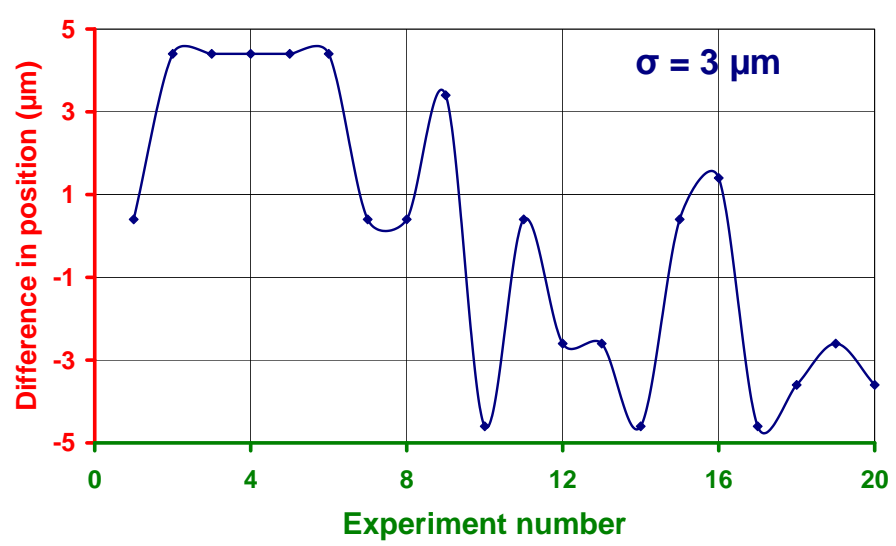

FIG. 2 Repeatability of self-focusing made on an aluminium mirror.

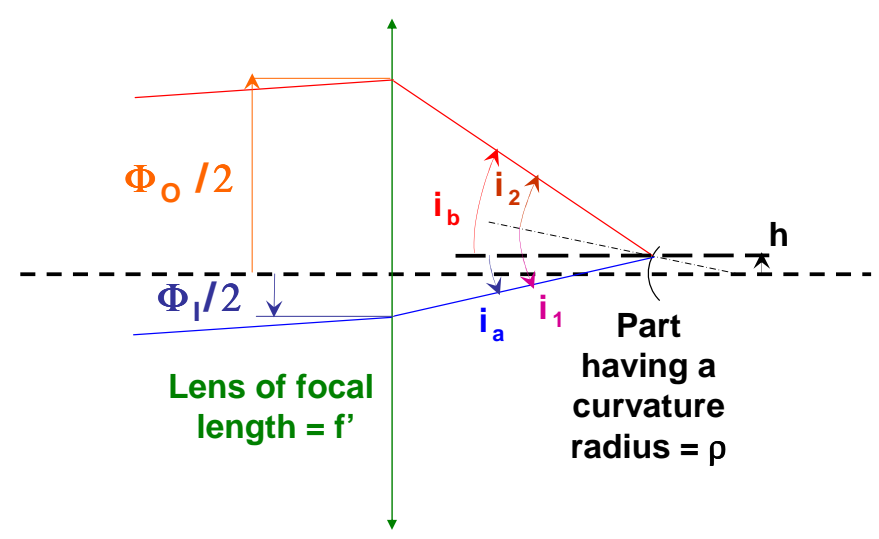

FIG. 3 Aim of the oversized condenser lens.

diameter to collect all the light (see Figure 3). Actually, if the condenser lens had smaller diameter, the reflected rays would be lost. This is not the case when lenses are oversized, so the reflectance measurement of the spherical or aspherical sample becomes possible.

According to Figure 3, we can deduce

$$
\rho=\frac{4 h f^{\prime}}{\phi_{\text {output }}-\phi_{\text {input }}}
$$

where $\rho$ is the curvature radius sample, $h$ is the radius of the image's spot onto the sample, $f^{\prime}$ is the focal length: $f^{\prime}=$ $38.5 \mathrm{~mm}, \phi_{\text {input }}$ is the diameter of entrance beam: $\phi_{\text {input }}=$ $17 \mathrm{~mm}$, and $\phi_{\text {output }}$ is the utile condenser diameter: $\phi_{\text {output }}=$ $46 \mathrm{~mm}$.

Following Eq. (1), it would be possible to measure optical components having a radius as small as $1 \mathrm{~mm}$.

A comparison with Zeiss Co. has already been made with an aspherical lens with antireflection coating having $38 \mathrm{~mm}$ focal length [9]. Herein, we present the results which have been obtained from several stainless steel ball bearings having a curvature radius from $22 \mathrm{~mm}$ to $7 \mathrm{~mm}$ therefore smaller than the previous aspherical lens [9], and two micro components. 


\section{INVESTIGATION WITH BALL BEARINGS}

These ball bearings are manufactured by SKF Co. The stainless steel of these balls is normally the same, so the reflectance must follow Fresnel laws (except for scattering due to manufacturing and local defects) whatever the diameter of the ball bearings. We are going to check and verify our reflectometer gives results which do not depend on the shape of the measured sample.

\subsection{Effect of the incidence angle on the measurement}

Before carrying out the various measurements, the effect of the incidence angle on the measurement is investigated. To determine this with our device, we measure the reflectance factor versus the incidence for a dielectric mirror at $633 \mathrm{~nm}$. Figure 4 shows the reflectance factor decreases less than $0.1 \%$ over a range of $6^{\circ}$.

If we want to measure the ball bearings accurately the beam must have an incidence of less than $3^{\circ}$. For a ball bearing having a $22 \mathrm{~mm}$ radius, we have calculated on a $3 \times 3 \mathrm{~mm}^{2}$ square the various beam incidences in degrees onto this ball. The results are given in Figure 5.

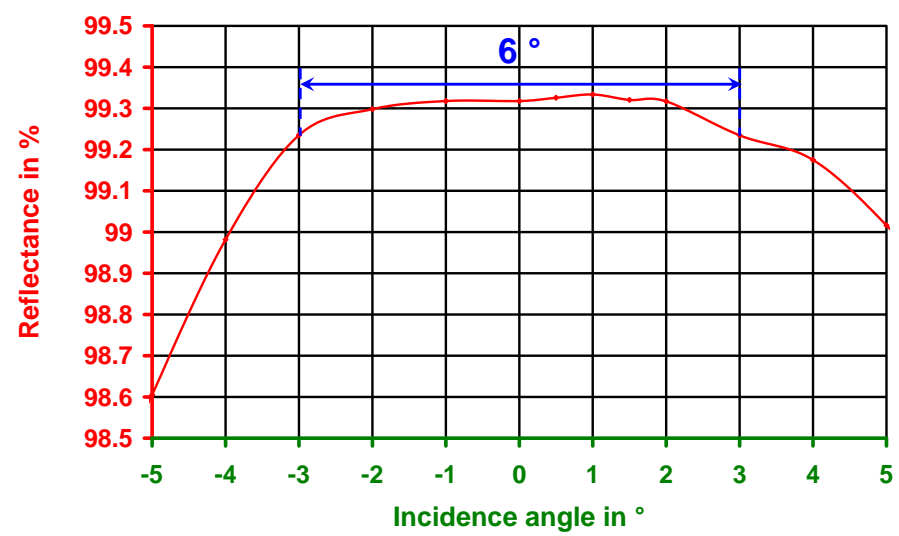

FIG. 4 Reflectance factor variation versus incidence angle.

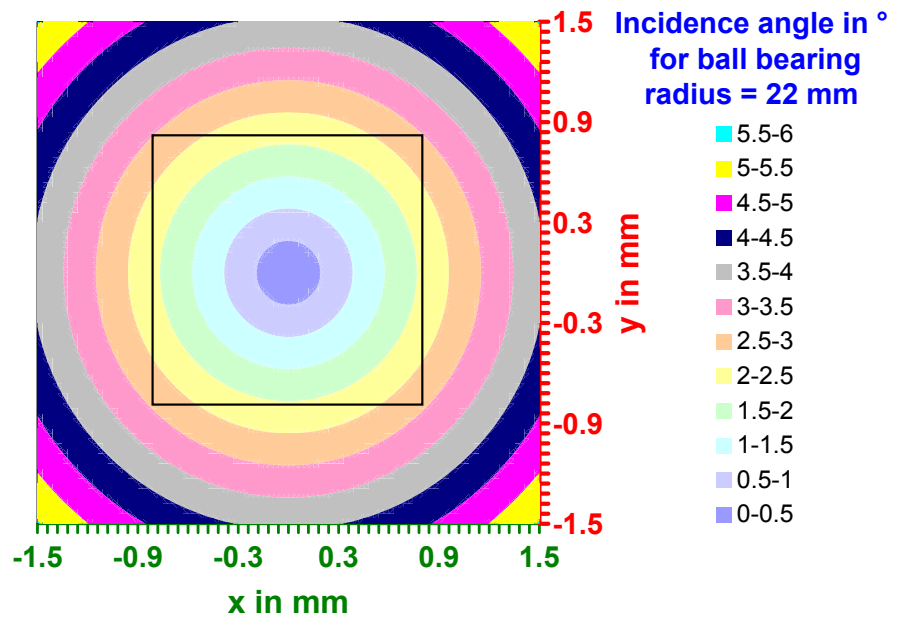

FIG. 5 Incidence mapping versus a $(x, y)$ spot location. The incidence is less than $3^{\circ}$ inside the black square.
To make a correct measurement, the spot location of the beam must be closer than $0.8 \mathrm{~mm}$ from the top of this ball.

Before each measurement, we carry out mapping at a wavelength, for example at $633 \mathrm{~nm}$, to find the ball top experimentally. Figure 6 shows a reflectance mapping on a $3 \times 3 \mathrm{~mm}^{2}$ area for the ball having a radius of $22 \mathrm{~mm}$.

For each ball bearing, we are going to measure the spectral reflectance at a point which is located inside the area (black square in Figure 6) where the reflectance factor appears to be constant. Of course, the size of this area depends on a ball bearing diameter.

\subsection{Experiment}

We have chosen eight ball bearings; we can see them in Figure 7 with a $1 €$ coin. Their respective diameters are $44.45 \mathrm{~mm}$, $28.57 \mathrm{~mm}, 23.80 \mathrm{~mm}, 20.63 \mathrm{~mm}, 20.61 \mathrm{~mm}, 19.05 \mathrm{~mm}$, $18.25 \mathrm{~mm}$, and $14.27 \mathrm{~mm}$.

These ball bearings have not been optically polished but machine-finished. We have put each ball bearing on a sample holder. Our software controls this sample holder in $x$, $y$, and $z$. For each ball bearing, we locate their top thanks to small mappings (see above, Section 3.1). We have chosen this site and have set it at the condenser focus by means of our software. During the spectral measurement, translating the sample holder along the optical axis for each wavelength conserves the optical conjugation. For each ball bearing, we have performed the measurement three times at the same site over the spectral range from $500 \mathrm{~nm}$ to $950 \mathrm{~nm}$. Below, in Fig-

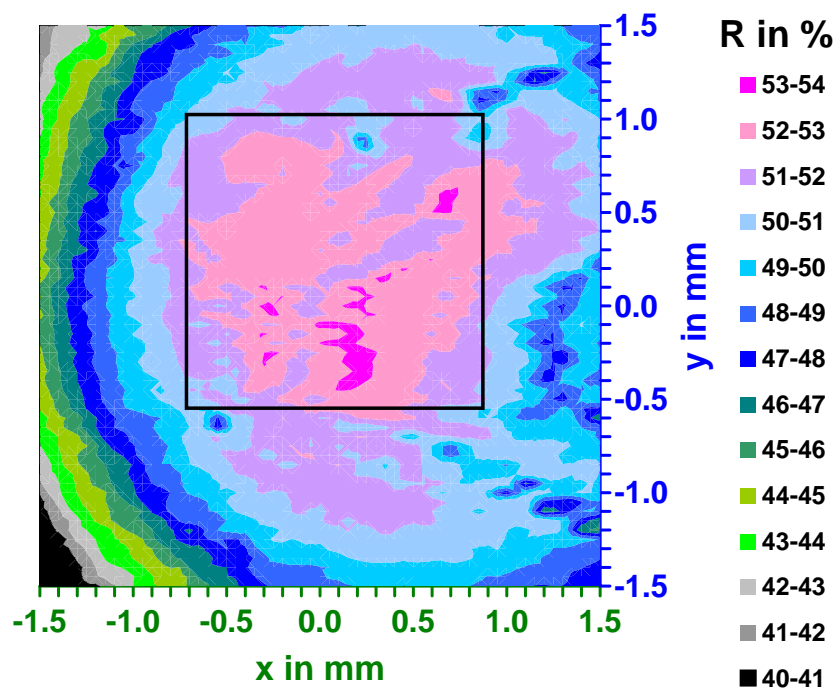

FIG. 6 Reflectance factor mapping at $633 \mathrm{~nm}$ for the ball bearing having a radius of $22 \mathrm{~mm}$.

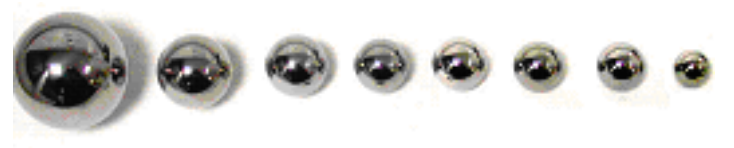

FIG. 7 Photograph of our eight ball bearings. 


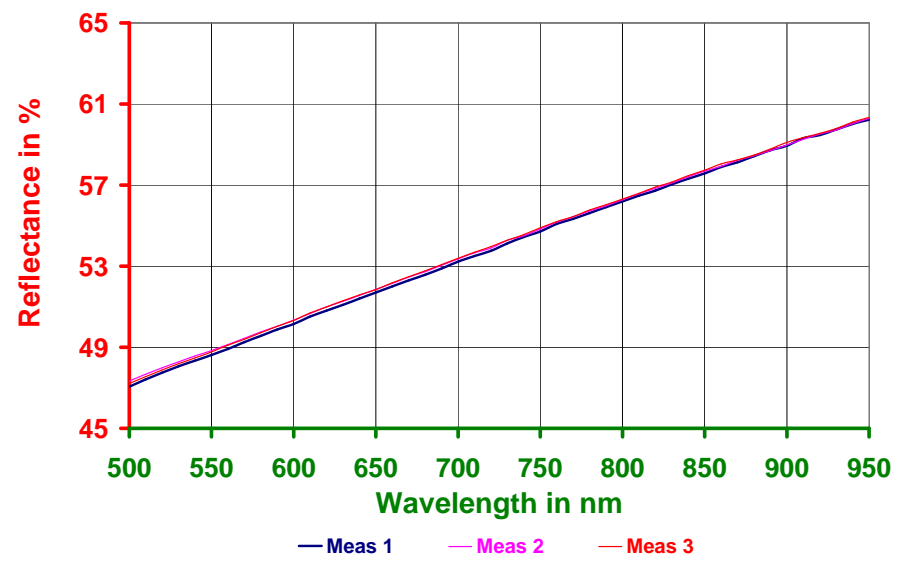

FIG. 8 Reflectance factor mapping at $633 \mathrm{~nm}$ for the ball bearing having a radius of $22 \mathrm{~mm}$.

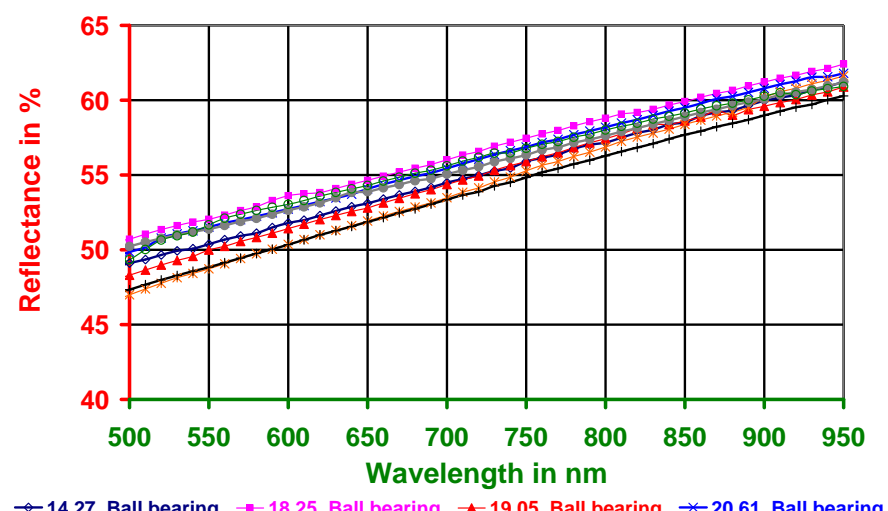

- 18.25 Ball bearing $₫ 19.05$ Ball bearing $* 20.61$ Ball bea

FIG. 9 Measurement of the eight ball bearings with our reflectometer.

ure 8 , we can see the three measurements obtained from the $44.45 \mathrm{~mm}$ diameter ball bearing.

The tiny difference between these three spectral measurements shows a good repeatability by the device. This repeatability is identical to that obtained with flat samples [2,8].

The eight measurements, one for each ball bearing, are given in Figure 9.

Table 1 gives the average reflectance over spectral range from 500 to $950 \mathrm{~nm}$ presented in ascending order with the respective diameter of the ball bearings.

\begin{tabular}{|c|c|c|}
\hline Sorting & $\begin{array}{c}\text { Average } \\
\text { reflectance } \\
(\%)\end{array}$ & $\begin{array}{c}\text { Ball bearing } \\
\text { diameter } \\
(\mathrm{mm})\end{array}$ \\
\hline 1 & 54.00 & 44.45 \\
\hline 2 & 54.38 & 20.63 \\
\hline 3 & 54.97 & 19.05 \\
\hline 4 & 55.16 & 14.27 \\
\hline 5 & 55.75 & 23.80 \\
\hline 6 & 56.01 & 28.57 \\
\hline 7 & 56.12 & 20.61 \\
\hline 8 & 56.68 & 18.25 \\
\hline
\end{tabular}

TABLE 1 Measurements made on the $44.45 \mathrm{~mm}$ diameter ball bearing.

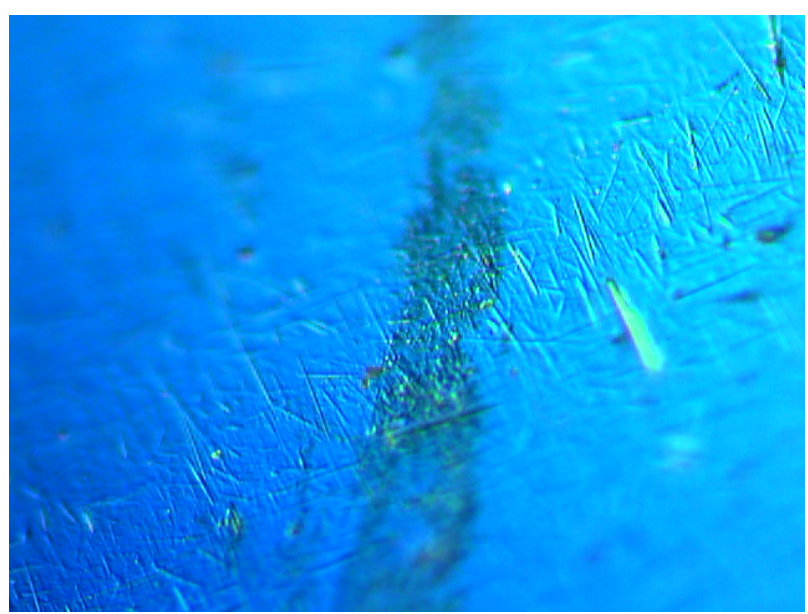

FIG. 10 Photograph of a ball bearing by a Normaski microscope.

We notice the results in reflectance obtained are similar (average reflectance over the spectral range from $500 \mathrm{~nm}$ to $950 \mathrm{~nm}$ is $55.4 \pm 1.4 \%$ ) for the eight ball bearings, even though they do not have the same radius (from $7.13 \mathrm{~mm}$ to $22.22 \mathrm{~mm}$ ). The curves are not placed in any particular order; they are mixed at random. This shows there is no link between the reflectance and the diameter of the ball bearings. First example, the curve of the ball bearing whose diameter is $19.05 \mathrm{~mm}$ is under the curve of the $18.25 \mathrm{~mm}$ ball bearing but also under the one whose diameter is $23.80 \mathrm{~mm}$. Second example, the two ball bearings which are close in diameter (the $20.61 \mathrm{~mm}$ and $20.63 \mathrm{~mm}$ diameter ball bearings) have a reflection coefficient comparatively remote (56.12\% and $54.38 \%$ respectively). They all fall within an interval of $3.7 \%$. For the shorter wavelengths the difference is $3.7 \%$ and for the larger wavelengths it is $2.1 \%$.

To explain the discrepancies observed, we have inspected them using Normaski interference optical microscopy (Leica/ DMRME microscope/ Germany) and can see they have a few local defects and scratches (see Figure 10).

As SKF Co. specifies on their website [12] that ball roughness is about $10 \mathrm{~nm}$, we have calculated the total integrated scattering (TIS) to estimate the level of the scattered light by the ball bearings due to their various roughnesses. The TIS is defined according to [13]

$$
\text { TIS }=\frac{\text { diffuse reflectance }}{\text { specular }+ \text { diffuse reflectance }}
$$

where the diffuse reflectance is the fraction of the incident beam which is reflected off-specular direction, and the specular reflectance is the fraction of the incident beam that is reflected into the specular direction. The relation between TIS [13] and rms roughness for a reflecting surface is given by

$$
\mathrm{TIS}=\left(\frac{4 \pi \delta}{\lambda}\right)^{2}
$$

where $\delta$ is the rms roughness of the surface and $\lambda$ the illuminating wavelength. Eq. (4) is for normal incidence illumination.

We calculate the fraction of scattered light variation versus wavelength over the spectral range ( $500 \mathrm{~nm}-950 \mathrm{~nm}$ ) by our 


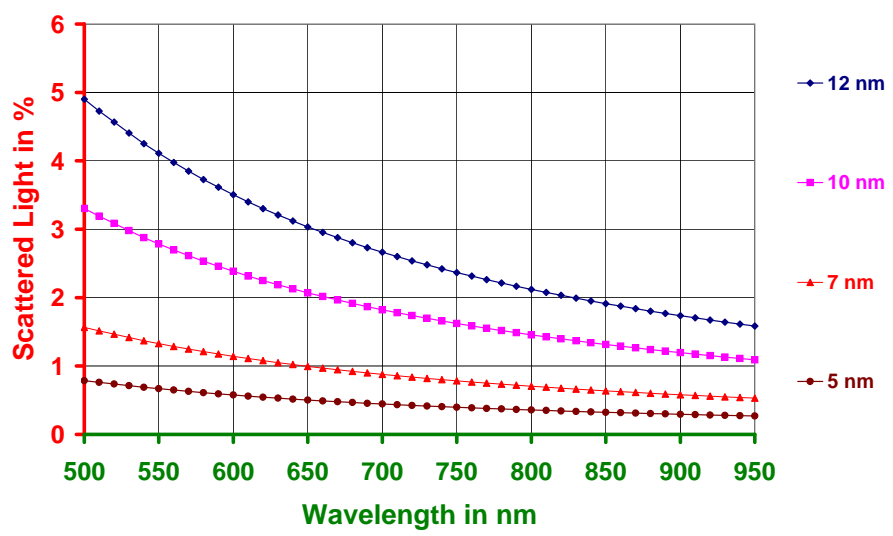

FIG. 11 Scattered light versus wavelength for four roughness values $(5 \mathrm{~nm}, 7 \mathrm{~nm}, 10 \mathrm{~nm}$ and $12 \mathrm{~nm}$ ).

ball bearings (average value) for four roughness values ( $5 \mathrm{~nm}$, $7 \mathrm{~nm}, 10 \mathrm{~nm}$, and $12 \mathrm{~nm}$ ). The results are given in Figure 11.

The tiny differences can be explained by the local defects, the various scratches, the various roughnesses and a local variation of composition, which have been observed by the Normaski optical microscope. If the ball bearings were optically polished, our results would be closer.

So we can conclude the reflectance measured with our device does not depend on the curvature radius of the sample. In fact, the size of the beam that is reflected by the sample is so small (about $100 \mu \mathrm{m}$ ) that the surface lit by the spot can be considered as flat. Therefore, we can say our reflectometer is able to determine the reflectance of pieces in form having curvature radii greater than to $7 \mathrm{~mm}$.

\section{FIRST MEASUREMENT OF MICROOPTICAL COMPONENTS}

Before this paper, many components (substrates, mirrors, antireflection coatings) have been measured by our reflectometer but these components had an area of a few $\mathrm{cm}^{2}$. Now, we present the first measurements carried out on small optical components.

\subsection{Plane component like a micro-mirror}

The small size of the spot on the tested component is about $100 \mu \mathrm{m}$ diameter. That enables to measure easily component heterogeneity [2], local defects [2, 8], and small mirrors with our measuring system. This potential use is highlighted by the measurements on three various sites of a protected metallic mirror. The measured micro-mirror, which is $2 \mathrm{~mm}$ diameter, has been bonded on a metallic rod (shown in Figure 12) and based on our adjustable holder. The mirror surface has been put at the collecting lens focus of our reflectometer by using motorized stages and our image processing.

The obtained curves in Figure 13 are similar for these three measurements. These curves have been fitted with a model compound by a metallic layer and a dielectric layer. The best fit corresponds to an aluminium layer coated by a silica film

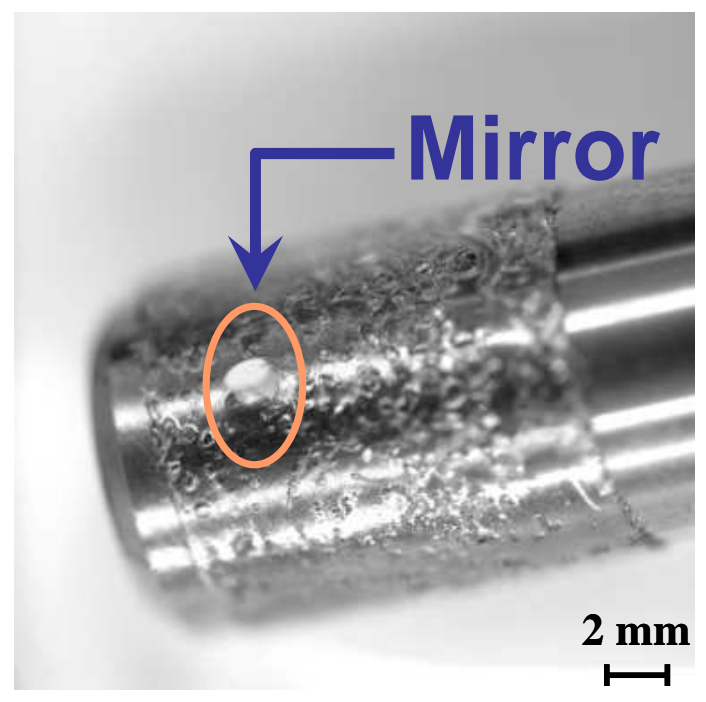

FIG. 12 Photograph of micro-mirror on its holder.

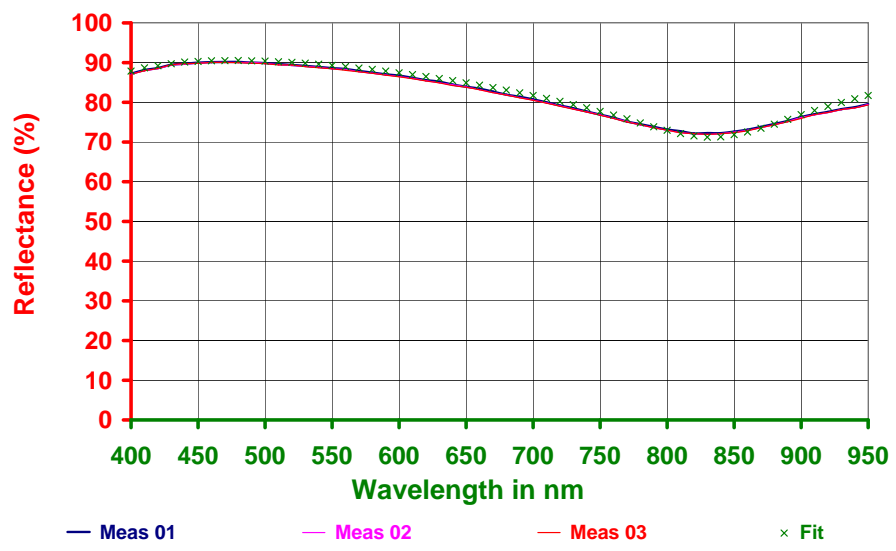

FIG. 13 The three spectral responses and the associated fit of the measured micromirror.

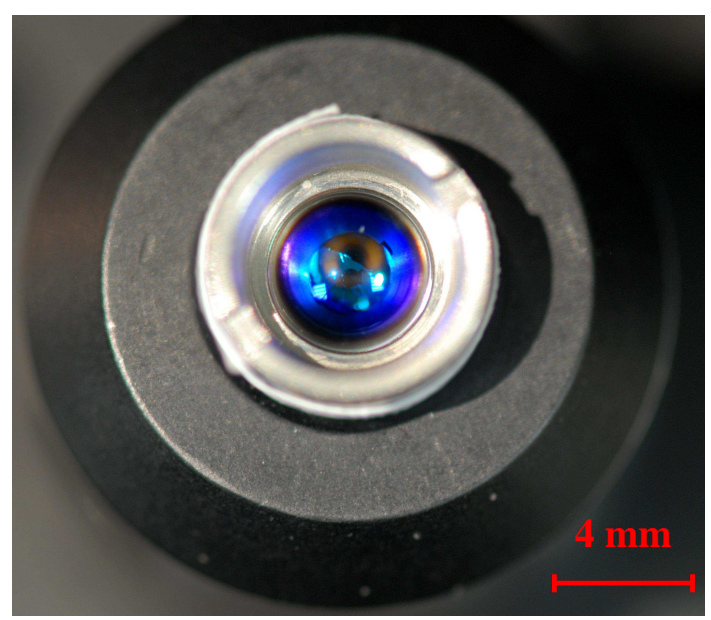

FIG. 14 Photograph of the measured micro lens.

having a thickness of $146 \mathrm{~nm}$. So, we can have an evaluation of the reflection on coatings on small optical components.

\subsection{Curved component like a lens}

This measured micro lens is supplied by Thorlabs Co. under the C440TME-B name [14] and shown in Figure 14. This 


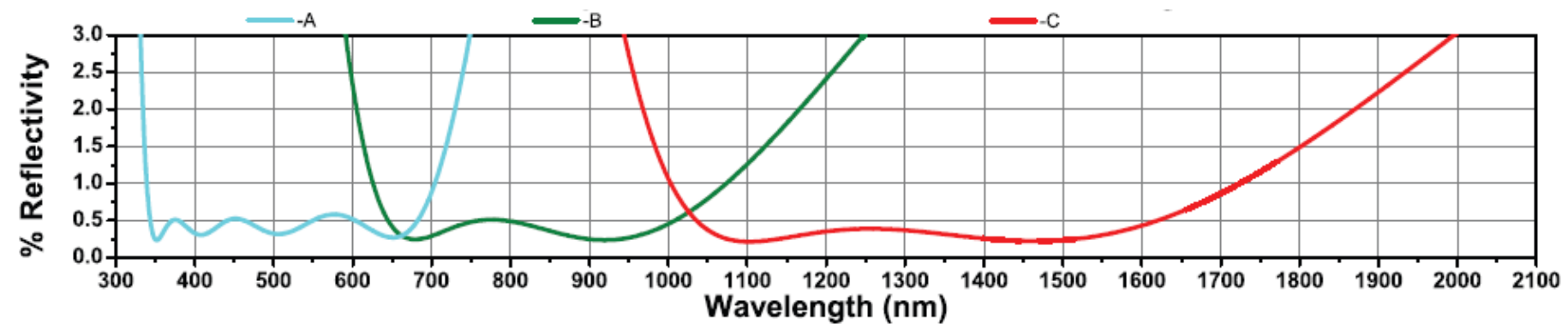

FIG. 15 Molded asphere broadband anti-reflection coating according to Thorlabs Co.; A: 350-700 nm, B: 650-1050 nm, and C:1050-1620 nm.

mounted lens is made from ECO-500 which is a glass with similar optical properties to C-0550, but does not contain hazardous materials. Its focal length is $2.95 \mathrm{~mm}$, its thickness is $4.06 \mathrm{~mm}$ and its diameter is $4.70 \mathrm{~mm}$. Both faces are aspherical. It is broadband antireflection coated from $600 \mathrm{~nm}$ to $1050 \mathrm{~nm}$ according to supplier (see green curve in Figure 15).

Its housing is mounted onto an adaptor plate which is based on our adjustable holder. The front face is put at the condenser lens focus of our reflectometer by using motorized stages and our image processing. We measure three times the coating response over the spectral range (400 nm-950 nm) onto a same site. These results are presented in Figure 16.

Our spectral responses are lightly different from the promised curve by the supplier (see Figure 15), but they seem similar to another antireflection coating of this supplier (see Figure 17).

We have checked this assumption by determining the design corresponding to the theoretical spectral response given in Figure 17. Using reverse engineering thin film homemade software based on a global optimization procedure derived from Tibor Csendes [15], we find a very good agreement with a 5-layer design alternating two materials of refractive index of $1.45 / 2.25$, possibly standing for Silica and Titanium dioxide refractive indexes. The thicknesses of the 5 layers are respectively of $54.7 \mathrm{~nm} / 25.5 \mathrm{~nm} / 56.7 \mathrm{~nm} / 184.6 \mathrm{~nm} / 133.7 \mathrm{~nm}$ from substrate to the air.

The manufacturing of such an antireflective surface undoubtfully leads to a higher level of residual reflectance, due to thickness and refractive index errors but also to the spectral

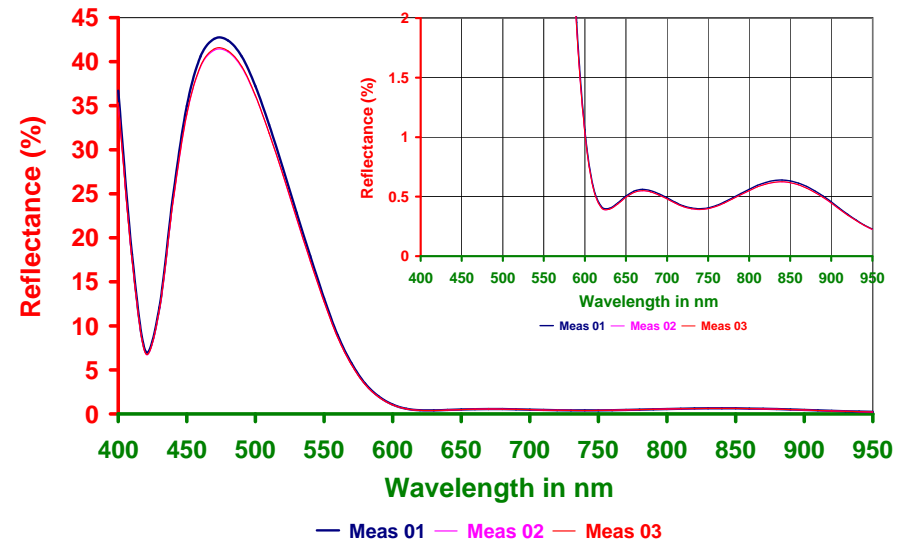

FIG. 16 Spectral response of C440TME-B lens measured by our reflectometer. dependence of the refractive index of the two materials. Classically, we can expect a 3\% error thickness tolerance and a 0.02 index variation from nominal values.

To check the influence of such errors, we have calculated the reflectance for more than 200 designs including random errors defined as above. In Figure 18, we have plotted 2 reflectance curves and an expected corridor of manufacturing. The blue curve corresponds to the theoretical 5-layer design (without errors). The red curve represents the spectral response of C440TME-B lens measured by our reflectometer. The area plotted in grey corresponds to the expected corridor of manufacturing: $80 \%$ of the 200 calculated designs including errors present a value of reflectance located inside this inter-

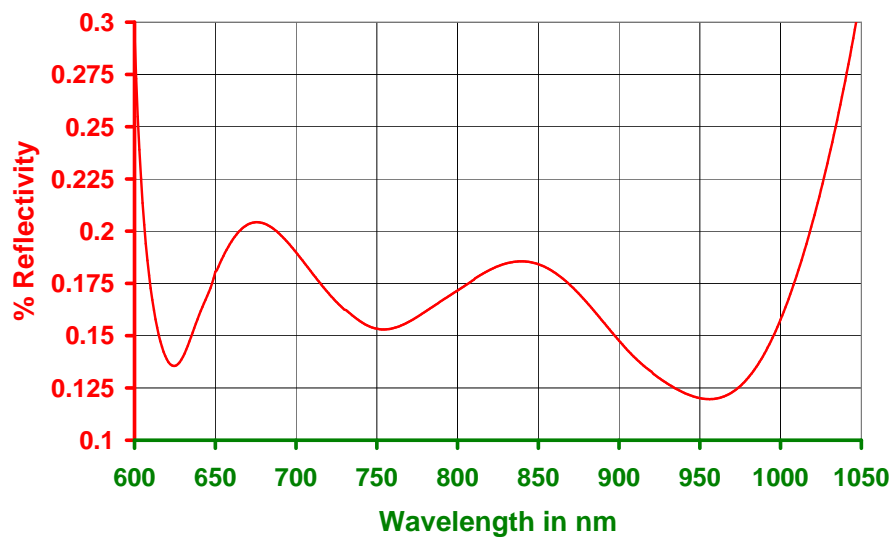

FIG. 17 600-1050 nm AR-coated calcite according to Thorlabs Co. [14].

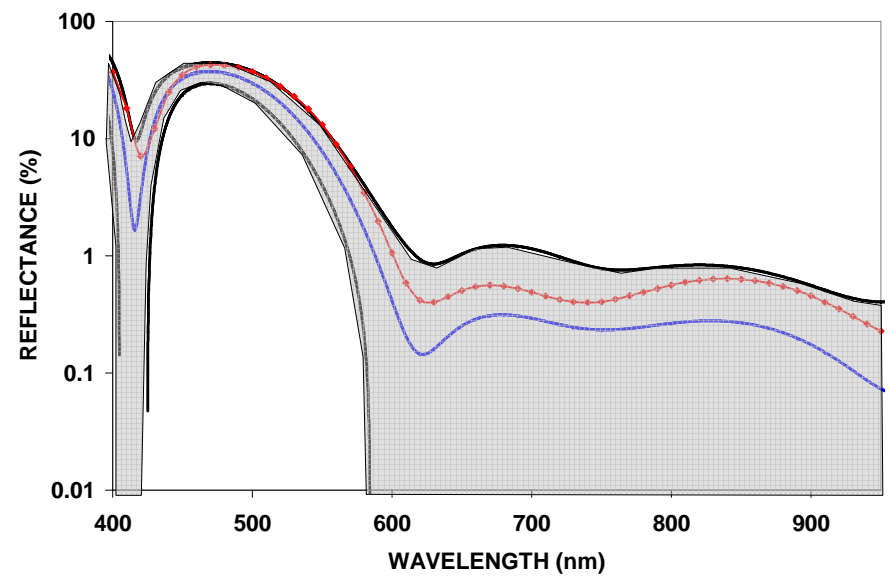

FIG. 18 Spectral reflectance for theoretical (blue curve) and experimental (red) designs. The gray region corresponds to a manufacturing expected corridor. 
val (we have just eliminated the $20 \%$ most unlikely values of reflectance).

As the experimental reflectance (red curve) is located inside the corridor for all wavelengths from $400 \mathrm{~nm}$ to $1050 \mathrm{~nm}$, the assumption of a C440TME-B lens coated by the supplier with the Figure 17 theoretical specificity is coherent.

We can conclude we could check the coating of this micro lens thanks to our reflectometer. In this case, the antireflection coating has not the promised specification by the supplier despite it replies to our request.

\section{CONCLUSIONS}

The obtained results on the balls show the measured reflectance does not depend on the radius curvature of the sample with our reflectometer when the curvature radius greater than $7 \mathrm{~mm}$. Our device provides access to a new metrology over a spectral range from $400 \mathrm{~nm}$ to $950 \mathrm{~nm}$ on complex components which was not available a few years ago.

The small size of the spot's reflectometer enables to measure small optical components.

We highlight we can also measure the antireflection coating on certain micro lenses. Nevertheless, we must assess evaluate for this kind of components the limits of our device with help from a manufacturer. Besides the curvature radius, we investigate the influence of component thickness. In the future, we could be involved to reduce the focal length of our collection lens in order to decrease the spot diameter and the deep of focus so as to measure smaller and thinner components.

\section{ACKNOWLEDGEMENTS}

We would like to acknowledge the SKF Company for having supplied the ball bearings which were used in these experiments, Xavier Le Borgne for photographs and Alan Gates and Annette Stansfeld for their help.

\section{References}

[1] http://physics.nist.gov/Divisions/Div844/facilities/specphoto/ facilities.html
[2] Ph. Voarino, H. Piombini, F. Sabary, D. Marteau, J. Dubard, J. Hameury, and J. R. Filtz, "High-accuracy measurements of the normal specular reflectance" Appl. 0pt. 47, C303-C309 (2008).

[3] G. J. Kopec, "Colorimetry as diagnostic tool in manufacture of antireflection coatings" Proc. SPIE 4517, 236-241 (2001).

[4] J. Schmoll, D. J. Robertson, C. M. Dubbeldam, F. Bortoletto, L. Pina, R. Hudec, E. Prieto, C. Norrie, and S. Ramsay-Howat, "Optical replication techniques for image slicers" New Astron. Rev. 50, 263-266 (2006).

[5] “Conferences and Meetings" Anal. Proc. 28, 234-239 (1991).

[6] H. Piombini, and Ph. Voarino, Dispositif et procédé de mesure de caractérisation par réflectométrie (PCT W02007/138032A1, 2007).

[7] X. Zheng, Z. Wang, and Y. Zhao, "An apparatus for the measurement of spectral specular reflectance of spot area in curved surfaces" Proc. SPIE 7156, 71560B (2008).

[8] H. Piombini, and Ph. Voarino, "Apparatus designed for very accurate measurement of the optical reflection" Appl. Opt. 46, 86098618 (2007).

[9] H. Piombini, S. Bruynooghe, and Ph. Voarino, "Spectral measurement in reflection on steeply aspheric surfaces" Proc. SPIE 7102, 71021 C (2008).

[10] J. Dubard, J. Hameury, J.-R. Filtz, D. Soler, Ph. Voarino, and H. Piombini, Normal reflectance measurement based on high reflectivity dielectric mirrors reference standard (10th International Conference on New Developments and Applications in Optical Radiometry, Daejon, 13-15 October 2008).

[11] H. Piombini, D. Soler, and Ph. Voarino, "New device to measure the reflectivity on steeply curved surface" Proc. SPIE 7018, 70181B (2008).

[12] http://www.skf.com/skf/support/html/dictionary/dictionary.jsp? lang=enctsite $=\mathrm{CH}$.

[13] J. M. Elson, J. P. Rahn, and J. M. Bennett, "Relationship of the total integrated scattering from multilayer-coated optics to angle of incidence, polarization, correlation length, and roughness crosscorrelation properties" Appl. Opt. 22, 3207-3219 (1983).

[14] Thorlabs Catalogue Volume 20 (2009).

[15] T. Csendes, "Nonlinear parameter estimation by global optimization - Efficiency and reliability" Acta Cybernetica 8, 361-370 (1988). 